\title{
Asymptotic Disturbance Attenuation Property Analysis for Discrete-Time Uncertain Switched Linear Systems
}

\author{
Hai Lin \\ Dept. of Electrical Engineering \\ University of Notre Dame \\ Notre Dame, IN 46556, USA
}

\author{
Guisheng Zhai \\ Dept. of Mechanical Engineering \\ Osaka Prefecture University \\ Sakai, Osaka 599-8531, Japan
}

\author{
Panos J. Antsaklis \\ Dept. of Electrical Engineering \\ University of Notre Dame \\ Notre Dame, IN 46556, USA
}

\begin{abstract}
In this paper, the disturbance attenuation properties in the sense of uniformly ultimate boundedness are investigated for a class of switched linear systems with parametric uncertainties and exterior disturbances. We aim to characterize the conditions under which the switched system can achieve a finite disturbance attenuation level. First, arbitrary switching signals are considered, and a necessary and sufficient condition is derived. Secondly, if the finite disturbance attenuation level is not preserved under arbitrary switchings, we restrict the switching signals so as to guarantee the finite disturbance attenuation levels. Two cases are considered here that depend on whether all the subsystems are uniformly ultimately bounded or not. For both cases, the switching signals are characterized by an average dwell time scheme. The techniques are based on multiple polyhedral Lyapunov and Lyapunov-like functions.
\end{abstract}

\section{INTRODUCTION}

A switched system is a dynamical system that consists of a finite number of subsystems described by differential or difference equations and a logical rule that orchestrates switching between these subsystems. Properties of this type of model have been studied for the past fifty years to consider engineering systems that contain relays and/or hysteresis. The last decade has seen an increasing research activities in the field of switched systems, and the main efforts typically focus on the analysis of dynamic behaviors, such as stability [13], [14], [9], [15], controllability and observability [4], [24] etc., and aim to design controllers with guaranteed stability and performance [3], [13]. However, the literature on robust performance of switched systems is still relatively sparse.

In this paper, we will focus on the disturbance attenuation analysis for classes of switched linear systems which are perturbed by both parameter variations and exterior disturbances. In particular, we consider a collection of discretetime linear systems described by the perturbed difference equations with parametric uncertainties

$$
x[k+1]=A_{q}(w) x[k]+E_{q} d[k], \quad k \in \mathbb{Z}^{+},
$$

where $q \in Q=\left\{q_{1}, q_{2}, \cdots, q_{N}\right\}$ and $\mathbb{Z}^{+}$stands for nonnegative integers. The state variable $x[k] \in \mathbb{R}^{n}$, disturbance input $d[k] \in \mathcal{D} \subset \mathbb{R}^{r}$. Assume that $\mathcal{D}$ is a $\mathrm{C}$-set. The term $\mathrm{C}$-set stands for a convex and compact set containing the origin in its interior. Let us assume polytopic uncertainty in

The partial support of the National Science Foundation (NSF CCR0113131) is gratefully acknowledged.

Corresponding author Hai Lin's e-mail: hlin1@nd. edu
(1), i.e., $A_{q}(w)=\sum_{j=1}^{v_{q}} w_{j} A_{q}^{j}, w_{j} \geq 0$ and $\sum_{j=1}^{v_{q}} w_{j}=$ 1. Notice that $A_{q}^{j}$ are known constant $n \times n$ matrices and the coefficients $w_{j}$ are unknown and possibly time varying. Without loss of generality, we assume that $E_{q} \in \mathbb{R}^{n \times r}$ is a constant matrix.

Combine the family of discrete-time uncertain linear systems (1) with a class of piecewise constant functions, $\sigma: \mathbb{Z}^{+} \rightarrow Q$. Then we can define the following timevarying system as a discrete-time switched linear system

$$
x[k+1]=A_{\sigma(k)}(w) x[k]+E_{\sigma(k)} d[k], \quad k \in \mathbb{Z}^{+}
$$

The signal $\sigma(k)$ is called a switching signal.

Because of parameter variations and exterior disturbances, it is only reasonable to expect that the trajectories of the switched system (2) converge into a neighborhood region of the equilibrium (the origin here), which is the so called practical stability or uniformly ultimate boundedness in the literature.

Definition 1: The uncertain switched system (2) under switching signal $\sigma(k)$ is Uniformly Ultimately Bounded $(U U B)$ if there exists a $\mathrm{C}$-set $\mathcal{S}$ such that for every initial condition $x(0)=x_{0}$, there exists $T\left(x_{0}\right)$ finite, and we have $x[k] \in \mathcal{S}$ for $k \geq T\left(x_{0}\right)$.

The disturbance attenuation properties considered here are in the sense of the uniformly ultimate boundedness. Given a collection of switching signals, if the switched system (2) is UUB for all these switching signals, then the switched system (2) is said to have finite disturbance attenuation level under this class of switching signals. If the switched system is UUB for all possible switching signals, the switched system (2) is said to have finite disturbance attenuation level under arbitrary switchings. We are also interested in characterizing a useful subclass of arbitrary switching signals such that the switched system (2) achieves finite disturbance attenuation level even when some of its subsystems do not have finite disturbance attenuation levels.

There are some related works in the literature on analyzing the disturbance attenuation properties of switched linear systems. For example, in [27], the $\mathcal{L}_{2}$ gain of continuoustime switched linear systems was studied using an average dwell time approach and piecewise quadratic Lyapunov functions, and the results were extended to discrete-time case in [28]. In [11], the root-mean-square (RMS) gain of a continuous-time switched linear system with slow switching was computed in terms of the solutions to a 
collection of Riccati equations. These robust performance problems considered are both in the signal's energy sense, and assume that the disturbances are constrained to have finite energy, i.e., bounded $\mathcal{L}_{2}$ norm. In practice, there are disturbances that do not satisfy this condition and act more or less continuously over time. Such disturbances are called persistent, and can not be treated in the above framework. In this paper, the disturbance attenuation property is in the signal's magnitude sense, i.e., time domain specifications. Moreover, we explicitly consider dynamic uncertainty in the switched system model here. Dynamics uncertainty in the plant model is one of the main challenges in control theory, and it is of practical importance to deal with dynamical uncertainties explicitly.

The persistent disturbance attenuation properties for uncertain hybrid/switched systems have been considered in our previous work [18], [19], [20]. In [18], the uniformly ultimate boundedness control problem was studied for both discrete-time and continuous-time uncertain switched linear systems. It was shown in [18] that, by proper switching, the closed-loop switched systems can reach a better disturbance attenuation level than any single subsystem does. The determination of optimal disturbance attenuation level, i.e., the $l_{\infty}$ induced gain, for uncertain switched systems and its decidability issue were discussed in [19] based on invariant set theory. The results for optimal disturbance attenuation level analysis were extended to classes of general uncertain hybrid systems in [20].

The problem studied in this paper is different from our previous work in the following aspects. First, here we consider asymptotic disturbance attenuation property for arbitrary initial conditions; in [19], [20] the disturbance attenuation property was in the $l_{\infty}$ induced gain sense and for proper initial conditions only. Secondly, the main objective of this paper is to derive conditions, on the subsystems' dynamics or the switching signals, under which the switched system (2) can achieve finite asymptotic disturbance attenuation level; in [18], the focus was on designing switching laws to achieve better asymptotic disturbance attenuation levels. Thirdly, our main methods here are based on polyhedral Lyapunov and Lyapunov-like functions, instead of set invariance theory as in [19], [20].

The rest of the paper is organized as follows. First, in Section II, the disturbance attenuation properties under arbitrary switching signals are considered. The aim is to identify necessary and sufficient conditions under which the switched system preserves uniformly ultimate boundedness under arbitrary switchings. Secondly, when the finite disturbance attenuation level is not preserved under arbitrary switchings, we turn to characterize the classes of switching signals to guarantee UUB. Two cases are considered for this problem that depend on whether all the subsystems are practically stable or not, which are developed in Section III and Section IV respectively.

Notation: The letters $\mathcal{E}, \mathcal{P}, \mathcal{S} \cdots$ denote sets, $\partial \mathcal{P}$ the boundary of set $\mathcal{P}$, and $\operatorname{int}\{\mathcal{P}\}$ its interior. For any real $\lambda \geq$
0 , the set $\lambda \mathcal{S}$ is defined as $\{x=\lambda y, y \in \mathcal{S}\}$. A polytope (bounded polyhedral set) $\mathcal{P}$ will be presented either by a set of linear inequalities $\mathcal{P}=\left\{x: F_{i} x \leq g_{i}, i=1, \cdots, s\right\}$, or by the dual representation in terms of the convex hull of its vertex set $\left\{x_{j}\right\}$, denoted by $\operatorname{Conv}\left\{x_{j}\right\}$. For $x \in \mathbb{R}^{n}$, the $l_{1}$ and $l_{\infty}$ norms are defined as $\|x\|_{1}=\sum_{i=1}^{n}\left|x_{i}\right|$ and $\|x\|_{\infty}=\max _{i}\left|x_{i}\right|$ respectively.

\section{Performance Under Arbitrary Switchings}

First, arbitrary switching signals are considered, when there is no a priori knowledge of the switching signals available. Clearly, it is necessary to assume that every subsystems has finite disturbance attenuation level. The interesting question is under what condition the switched linear system (2) achieves a finite asymptotic disturbance attenuation level, i.e., uniformly ultimate boundedness.

It was shown in [7] that a linear time-variant systems is UUB if and only if the corresponding autonomous system is asymptotically stable. Therefore, this problem is transformed into a stability analysis problem for autonomous switched system under arbitrary switchings, which has been studied in the literature extensively, see for example the survey papers [14], [9], the recent book [15] and the references cited therein. This problem is typically being dealt with by constructing a common Lyapunov function. For example, various approaches have been presented [22], [23], [16], [17] to find a common quadratic Lyapunov function for the family of systems, ensuring the asymptotic stability of switched systems for any switching signal. In [16] and [2], Lie algebra conditions were given, which imply the existence of a common quadratic Lyapunov function. It is worth pointing out that a converse Lyapunov theorem was derived in [8] for the globally asymptotic stability of arbitrary switching systems. This converse Lyapunov theorem justifies the common Lyapunov function method which was pursued in the literature. However, most of the work was restricted to the case of quadratic Lyapunov function, which only gave sufficient stability test criteria.

\section{A. Necessary and Sufficient Condition}

In this section, we will give a necessary and sufficient condition for asymptotic stability of switched linear systems with arbitrary switchings. For such purpose, let us first introduce a technical lemma [1] for the robust stability of linear time-variant systems

$$
x[k+1]=A(k) x[k]
$$

where $A(k) \in \mathcal{A} \hat{=} \operatorname{Conv}\left\{A_{q_{1}}^{1}, A_{q_{1}}^{2}, \cdots, A_{q_{1}}^{v_{q_{1}}}, A_{q_{2}}^{1}, \cdots\right.$, $\left.A_{q_{2}}^{v_{q_{2}}}, \cdots, A_{q_{N}}^{1}, \cdots, A_{q_{N}}^{v_{q_{N}}}\right\}$. In other words, the state matrix $A(k)$ of the above linear time-variant system (3) is constructed by a convex combinations of all the subsystems' vertex matrices of the switched linear system (2). A necessary and sufficient condition [1] for the asymptotic stability of the above linear time-variant system (3) is stated as follows. 
Lemma 1: The polytopic uncertain linear time-variant system (3) is globally asymptotically stable if and only if there exists a finite integer $\mathbf{n}$ such that $\left\|A_{i_{1}} A_{i_{2}} \cdots A_{i_{\mathbf{n}}}\right\|<$ 1 for all n-tuple $A_{i_{j}} \in \operatorname{vert}\{\mathcal{A}\}=\left\{A_{q_{1}}^{1}, A_{q_{1}}^{2}, \cdots, A_{q_{1}}^{v_{q_{1}}}\right.$, $\left.A_{q_{2}}^{1}, \cdots, A_{q_{2}}^{v_{q_{2}}}, \cdots, A_{q_{N}}^{1}, \cdots, A_{q_{N}}^{v_{q_{N}}}\right\}$, with $j=1, \cdots, \mathbf{n}$.

Here the norm $\|\cdot\|$ stands for either 1 norm or $\infty$ norm of a matrix. Asymptotic stability of the switched systems can be expressed as the following proposition. The proofs are omitted here due to space limit.

Proposition 1: The switched linear system $x[k+1]=$ $A_{\sigma(k)}(w) x[k]$, where $A_{\sigma(k)}(w) \in\left\{A_{q_{1}}(w), A_{q_{2}}(w), \cdots\right.$, $\left.A_{q_{N}}(w)\right\}$, is globally asymptotically stable under arbitrary switchings if and only if there exists a finite integer $\mathbf{n}$ such that

$$
\left\|A_{i_{1}} A_{i_{2}} \cdots A_{i_{\mathbf{n}}}\right\|<1,
$$

for all n-tuple $A_{i_{j}} \in \operatorname{vert}\{\mathcal{A}\}=\left\{A_{q_{1}}^{1}, A_{q_{1}}^{2}, \cdots, A_{q_{1}}^{v_{q_{1}}}\right.$, $\left.A_{q_{2}}^{1}, \cdots, A_{q_{2}}^{v_{q_{2}}}, \cdots, A_{q_{N}}^{1}, \cdots, A_{q_{N}}^{v_{q_{N}}}\right\}$, with $j=1, \cdots, \mathbf{n}$.

From the above proposition, we obtain a necessary and sufficient condition for the switched systems to achieve a finite disturbance attenuation level under arbitrary switchings as stated in the following corollary.

Corollary 1: Under the assumption that each subsystem has finite disturbance attenuation level, the switched linear system (2) also achieves a finite disturbance attenuation level under arbitrary switchings if and only if there exists a finite integer $\mathbf{n}$ such that

$$
\left\|A_{i_{1}} A_{i_{2}} \cdots A_{i_{\mathbf{n}}}\right\|<1
$$

for all $\mathbf{n}$-tuple $A_{i_{j}} \in \operatorname{vert}\{\mathcal{A}\}$, with $j=1, \cdots, \mathbf{n}$.

\section{B. Equivalence}

As a byproduct, we prove the equivalence between the robust globally asymptotic stability for polytopic uncertain linear time-variant systems and the globally asymptotic stability for switched linear systems under arbitrary switchings. The equivalence can be expressed as the following corollary.

Corollary 2: The global asymptotic stability for polytopic uncertain linear time-variant systems $x[k+1]=$ $A(k) x[k]$, where $A(k) \in \mathcal{A}$, is equivalent to the global asymptotic stability for arbitrary switching systems $x[k+$ $1]=A_{\sigma(k)}(w) x[k]$.

It is interesting that the robust stability of a polytopic uncertain linear time-variant system, which has infinite number of possible dynamics (modes), is equivalent to only considering a finite number of its vertex dynamics as an arbitrary switching system. Although we only prove the equivalence in the discrete-time case, this result is also true in the continuous-time case. This fact bridges two originally distinct research fields. Therefore, existing results in the robust stability area, which has been extensively studied for over two decades, can be directly introduced to study the arbitrarily switching systems and vice versa.

\section{Performance Under Slow Switchings: UUB SUBSYSTEMS}

If the finite disturbance attenuation level is not preserved under arbitrary switching signals, it is still possible to restrict the switching signals so as to achieve a finite disturbance attenuation level. It is shown in [10], [27], [12] that the stability and performance could be preserved under certain constrained switchings, for example slow switching with bounded average dwell time. Therefore, it is important to classify the classes of switching signals under which the switched system remains stable. The stability analysis with constrained switchings has been usually pursued in the framework of multiple Lyapunov functions (MLF), see for example [25], [26], [9], [14], [15] and references therein.

In this section, it is assumed that each subsystem is UUB along with a Lyapunov function. We will employ multiple polyhedral Lyapunov functions in this section to characterize switching laws such that the switched system (2) is uniformly ultimate bounded.

\section{A. Set-Induced Lyapunov Function}

Following the notation of [5], we call a function $\Psi$ : $\mathbb{R}^{n} \rightarrow \mathbb{R}$ a gauge function if $\Psi(x) \geq 0, \Psi(x)=0 \Leftrightarrow$ $x=0$; for $\mu>0, \Psi(\mu x)=\mu \Psi(x)$; and $\Psi(x+y) \leq$ $\Psi(x)+\Psi(y), \forall x, y \in \mathbb{R}^{n}$. A gauge function is convex and it defines a distance of $x$ from the origin which is linear in any direction. If $\Psi$ is a gauge function, we define the closed set (possibly empty) $\bar{N}[\Psi, \xi]=\left\{x \in \mathbb{R}^{n}: \Psi(x) \leq \xi\right\}$. It is easy to show that the set $\bar{N}[\Psi, \xi]$ is a C-set for all $\xi>0$. On the other hand, any C-set $\mathcal{S}$ induces a gauge function $\Psi_{\mathcal{S}}(x)$ (Known as Minkowski function of $\mathcal{S}$ ), which is defined as $\Psi(x) \doteq \inf \{\mu>0: x \in \mu \mathcal{S}\}$. Therefore a C-set $\mathcal{S}$ can be thought of as the unit ball $\mathcal{S}=\bar{N}[\Psi, 1]$ of a gauge function $\Psi$ and $x \in \mathcal{S} \Leftrightarrow \Psi(x) \leq 1$.

Consider the discrete-time uncertain linear subsystem:

$$
x[k+1]=A(w) x[k]+E d[k]
$$

Note that the subscript $q$ is dropped in this subsection for notational simplicity.

First, we give a definition of the Lyapunov function. In this case, a Lyapunov function outside $\mathcal{S}$ for system (6) can be defined as a continuous function $\Psi: \mathbb{R}^{n} \rightarrow \mathbb{R}^{+}$such that $N[\Psi, \kappa] \subset \mathcal{S}$, for some positive scalar $\kappa$, for which the following conditions hold:

if $x \notin N[\Psi, \kappa]$ then there exists $\beta>0$ such that

$$
\Psi(A(w) x+E d)-\Psi(x) \leq-\beta ;
$$

if $x \in N[\Psi, \kappa]$ then

$$
\Psi(A(w) x+E d) \leq \kappa .
$$

Lemma 2: [6] If there exists a a Lyapunov function outside $\mathcal{S}$ for system (6), then it is uniformly ultimately bounded (UUB) in $\mathcal{S}$.

It was shown in [6] that if a Lyapunov function exists that solves the uniform ultimate boundedness problem in 
a certain convex neighborhood of the origin for system (6), then there exists a polyhedral Lyapunov function that solves the problem in the same neighborhood. Therefore, without loss of generality, we assume that system (6) has a polyhedral Lyapunov function $\Psi$ outside $\mathcal{S}$. The next question is how to compute the polyhedral Lyapunov function $\Psi(\cdot)$.

It is easy to derive from the definition of the Lyapunov function $\Psi$ that

$$
\Psi(x[k]) \leq \max \left\{\lambda^{t} \Psi(x[0]), 1\right\}
$$

for some $\lambda$ with $0<\lambda<1$. This property motivates the following concept of contractive set.

Definition 2: Given $\lambda, 0<\lambda<1$, a set $\mathcal{S}$ is said $\lambda$ contractive with respect to system (6), if for any $x \in \mathcal{S}$, $\operatorname{post}(x, \mathcal{W}, \mathcal{D}) \subseteq \lambda \mathcal{S}$. Here post $(\cdot)$ is defined as

$\operatorname{post}(x, \mathcal{W}, \mathcal{D})=\left\{x^{\prime}: x^{\prime}=A(w) x+E d ; \forall w \in \mathcal{W}, d \in \mathcal{D}\right\}$

which represents all the possible next step states from the current state $x$.

Let $\mathcal{S}$ be an assigned C-set in $\mathbb{R}^{n}$. We say that a $\lambda$ contractive set $\mathcal{P}_{m} \subseteq \mathcal{S}$ is maximal in $\mathcal{S}$ if and only if every $\lambda$-contractive set $\mathcal{P}$ contained in $\mathcal{S}$ is also contained in $\mathcal{P}_{m}$.

Consider the following sequence of sets:

$$
\left\{\mathcal{X}_{k}\right\}: \mathcal{X}_{0}=\mathcal{S}, \mathcal{X}_{k}=\operatorname{pre}\left(\lambda \mathcal{X}_{k-1}\right) \cap \mathcal{S} ; \quad k=1,2, \cdots
$$

where $\operatorname{pre}(\mathcal{S})$ is defined as

$$
\operatorname{pre}(\mathcal{S})=\left\{x \in \mathbb{R}^{n}: \operatorname{post}(x, \mathcal{W}, \mathcal{D}) \subseteq \mathcal{S}\right\}
$$

Then the maximal $\lambda$-contractive set $\mathcal{P}_{m} \subseteq \mathcal{S}$ is given by $\mathcal{P}_{\lambda}=\bigcap_{k=0}^{\infty} \mathcal{X}_{k}$.

Proposition 2: If $\mathcal{P}_{\lambda}=\bigcap_{k=0}^{\infty} \mathcal{X}_{k}$ is nonempty, then subsystem (6) is uniformly ultimately bounded (UUB) in $\mathcal{P}_{\lambda} \subseteq \mathcal{S}$.

It is easy to show that nonempty $\mathcal{P}_{\lambda}$ is a C-set, whose induced Minkowski functional $\Psi_{\mathcal{P}_{\lambda}}(x)$ serves as a Lyapunov function outside the target set $\mathcal{S}$. Such Lyapunov function is uniquely generated from the target set $\mathcal{S}$ for any fixed $\lambda$, so it is named Set-induced Lyapunov Function (SILF) in the literature, see [5], [6] and its references. For systems with linearly constrained uncertainties, it can be shown that such function may be derived by numerically efficient algorithms involving polyhedral sets.

\section{B. UUB Analysis}

It is assumed that each subsystem is UUB with decay rate $\lambda_{q}$ along with a polyhedral Lyapunov function, $\Psi_{q}(\cdot)$. Now, define the multiple Lyapunov function candidate as

$$
V(x[k])=\Psi_{\sigma(k)}(x[k])
$$

Let $k_{1}, k_{2}, \cdots$ stand for the time points at which switching occurs, and write $q_{j}$ for the value of $\sigma(k)$ on $\left[k_{j-1}, k_{j}\right)$. Then, for any $k$ satisfying $k_{0}=0<\cdots<$ $k_{i} \leq k<k_{i+1}$, we obtain

$$
V(x[k]) \leq \max \left\{\lambda_{q_{i}}^{k-k_{i}} \Psi_{q_{i}}\left(x\left[k_{i}\right]\right), 1\right\}
$$

Also, there exists a constant scalar $\mu$ such that $\Psi_{i}(x) \leq$ $\mu \Psi_{j}(x)$ and $\Psi_{j}(x) \leq \mu \Psi_{i}(x)$, for all $x \in \mathbb{R}^{n}$. A possible choice for $\mu$ is the largest value among $\Psi_{j}\left(v_{j}\right), \forall v_{j} \in$ $\operatorname{vert}\left\{N\left[\Psi_{i}, 1\right]\right\}$, and $\Psi_{i}\left(v_{j}\right), \forall v_{j} \in \operatorname{vert}\left\{N\left[\Psi_{j}, 1\right]\right\}$. This can be straightforwardly verified by exploring the geometric property of the level sets of $\Psi_{i}(x)$ and $\Psi_{j}(x)$.

Denote $\lambda_{0}=\max _{q \in Q}\left\{\lambda_{q}\right\}$. Then

$$
\begin{aligned}
V(x[k]) & \leq \max \left\{\lambda_{0}^{k-k_{i}} \Psi_{q_{i}}\left(x\left[k_{i}\right]\right), 1\right\} \\
& \leq \max \left\{\lambda_{0}^{k-k_{i-1}} \mu \Psi_{q_{i-1}}\left(x\left[k_{i}\right]\right), 1\right\} \\
& \leq \cdots \\
& \leq \max \left\{\lambda_{0}^{k} \mu^{i} \Psi_{q_{0}}(x[0]), 1\right\} \\
& =\max \left\{\lambda_{0}^{k} \mu^{N_{\sigma}(k)} V(x[0]), 1\right\}
\end{aligned}
$$

where $N_{\sigma}(k)$ denotes the number of switchings of $\sigma(k)$ over the interval $[0, k)$. Assume that there exists a scalar $0<\lambda^{*}<1$ such that

$$
\lambda_{0}^{k} \mu^{N_{\sigma}(k)} \leq\left(\lambda^{*}\right)^{k}
$$

This inequality is equivalent to

$$
N_{\sigma}(k) \leq \frac{k}{\tau_{a}^{*}}, \quad \tau_{a}^{*}=\frac{\ln \mu}{\ln \lambda^{*}-\ln \lambda_{0}}
$$

which is exactly an average dwell time scheme. The constant $\tau_{a}^{*}$ is called the average dwell time. The idea is that there may exist consecutive switching separated by less than $\tau_{a}^{*}$, but the average time interval between consecutive switchings is not less than $\tau_{a}^{*}$. Note that the concept of average dwell time between subsystems was originally proposed for continuous-time switched systems in [10].

From the average dwell switching scheme, we obtain

$$
V(x[k]) \leq \max \left\{\left(\lambda^{*}\right)^{k} V(x[0]), 1\right\} .
$$

This implies that the entire system is UUB. In summary, we have

Theorem 1: If all the subsystems of switched system (2) are UUB, then the switched system (2) achieves a finite asymptotic disturbance attenuation level under switching signals with average dwell time no less than $\frac{\ln \mu}{\ln \lambda^{*}-\ln \lambda_{0}}$ in the sense of (10).

\section{Performance Under Slow Switchings: With NON-UUB SUBSYSTEMS}

In the previous section, we specified a class of slow switching signals that guarantee the uniformly ultimate boundedness for uncertain switched linear systems (2) with stable subsystems. However, there are some cases that it is unavoidable to switch to unstable subsystems, such as controller failure in fault tolerant systems, packet dropouts in networked control systems etc.

In this section, we will study the case when not all the subsystems are uniformly ultimately bounded. Without lost of generality, it is assumed that the first $r$ subsystem are UUB along with a Lyapunov function, while the rest subsystems are not UUB. To make the problem tractable, the expansion rates of these unstable subsystems are limited. 
In particular, we assume that the expansion of the unstable subsystems are bounded in the sense of set-induced Lyapunov-like functions, which is introduced below and represents an extension of classical set-induced Lyapunov functions.

\section{A. Set-Induced Lyapunov-like Functions}

For the subsystem that is not UUB, there does not exist set-induced Lyapunov function as derived in the previous section. Therefore, we generalize the concept of $\lambda$-contractive set and derive a set-induced Lyapunov-like function. For such purpose, we first introduce the following definition for a Lyapunov-like function.

A Lyapunov-like function outside $\mathcal{S}$ for subsystem (6) can be defined as a continuous function $\Psi: \mathbb{R}^{n} \rightarrow \mathbb{R}^{+}$such that $N[\Psi, \kappa] \subset \mathcal{S}$, for some positive scalar $\kappa$, for which the following conditions hold:

if $x \notin N[\Psi, \kappa]$ then there exists $\lambda \geq 1$ such that

$$
\Psi(A(w) x+E d) \leq \lambda \Psi(x)
$$

if $x \in N[\Psi, \kappa]$ then

$$
\Psi(A(w) x+E d) \leq \lambda \kappa .
$$

This Lyapunov-like function outside $\mathcal{S}$ definition is quite similar to the definition of Lyapunov function outside $\mathcal{S}$ in the previous section. The difference here is that the value of a Lyapunov-like function increases at every step instead of decreasing. To capture this trend of expansion in the state space, we introduce the following expansive set definition, which is a counter-part to contractive set in the previous section.

Definition 3: Given $\lambda, \lambda>1$, a set $\mathcal{S}$ is said $\lambda$-expansive with respect to subsystem (6), if for any $x \in \mathcal{S}$ such that $\operatorname{post}(x, \mathcal{W}, \mathcal{D}) \subseteq \lambda \mathcal{S}$.

Definition 4: Subsystem (6) is said to have expansive index $\lambda>1$ to the $\mathrm{C}$-set $\mathcal{S}$ iff there exists a gauge function $\Psi(x)$ and a constant $\xi>0$ such that the ball $\bar{N}[\Psi, \xi] \subseteq \mathcal{S}$ and, if $x \notin \operatorname{int}\{\bar{N}[\Psi, \xi]\}$, then $\Psi(\operatorname{post}(x, w, d)) \leq \lambda \Psi(x)$ for all $w \in \mathcal{W}$ and $d \in \mathcal{D}$ (or. equivalently, $\bar{N}[\Psi, \mu]$ is $\lambda$-expansive for all $\mu \geq \xi$ ).

Intuitively, the concepts of $\lambda$-expansive set and expansive index $\lambda$ reflect how explosive the unstable subsystems are. For LTI subsystems, this is released to the magnitude of their unstable eigenvalues.

The next question is how to determine such $\lambda$-expansive set for an unstable subsystem. It turns out that the procedure developed for contractive sets can be extended to expansive set in parallel, which is described in the following.

Let $\mathcal{S}$ be assigned. We say that a $\lambda$-expansive set $\mathcal{P}_{\lambda} \subseteq \mathcal{S}$ is maximal in $\mathcal{S}$ iff every $\lambda$-expansive set $\mathcal{P}$ contained in $\mathcal{S}$ is also contained in $\mathcal{P}_{\lambda}$. Consider the following sequence of sets:

$$
\left\{\mathcal{X}_{k}\right\}: \mathcal{X}_{0}=\mathcal{S}, \quad \mathcal{X}_{k}=\operatorname{pre}\left(\lambda \mathcal{X}_{k-1}\right) \cap \mathcal{S} ; \quad k=1,2, \cdots
$$

Proposition 3: The maximal $\lambda$-expansive set $\mathcal{P}_{\lambda} \subseteq \mathcal{S}$ is given by $\mathcal{P}_{\lambda}=\bigcap_{k=0}^{\infty} \mathcal{X}_{k}$.
The above iterative procedure for determining the maximal $\lambda$-expansive set may fail to terminate in finite steps. However, under certain conditions, the maximal $\lambda$ expansive set $\mathcal{P}_{\lambda}$ could be determined by finite iterations as shown below.

Proposition 4: Assume that $\mathcal{P}_{\lambda}$ is a C-set, and $\lambda>1$. Then for every $\lambda^{*}$ such that $1<\lambda<\lambda^{*}$, there exists $\mathbf{k}$ such that $\mathcal{X}_{k}$ is $\lambda^{*}$-expansive for all $k \geq \mathbf{k}$.

It is straightforward to show that a $\bar{\lambda}$-expansive set has the following property, just like a $\lambda$-contractive set.

Proposition 5: If $\mathcal{P}$ is $\lambda$-expansive set for system (6), then $\mu \mathcal{P}$ is so for all $\mu \geq 1$. (if $\mathcal{D}=\{0\}$, for all $\mu \geq 0$.)

With the existence and determination of a non-empty maximal $\lambda$-expansive set $\mathcal{P}_{\lambda} \subseteq \mathcal{S}$, we may induce a Lyapunov-like function from $\mathcal{P}_{\lambda}$.

Proposition 6: If $\mathcal{P}_{\lambda}=\bigcap_{k=0}^{\infty} \mathcal{X}_{k}$ is nonempty, then $\mathcal{P}_{\lambda}$ is a C-set and its Minkowski functional $\psi(x)=\Psi_{\mathcal{P}_{\lambda}}(x)$ is a Lyapunov-like function for system (6) outside $\mathcal{P}_{\lambda} \subseteq \mathcal{S}$.

\section{B. UUB Analysis}

For any switching signal $\sigma(k)$ and any $k>0$, let $K_{i}(k)$ denote the total period that the $q_{i}$-th subsystem is activated during $[0, k)$. Define $K^{-}(k)=\sum_{i<r, q_{i} \in Q} K_{i}(k)$, which stands for the total activation period of the UUB subsystems. On the other hand, $K^{+}(k)=\sum_{i>r, q_{i} \in Q} K_{i}(k)$ denotes the total activation period of the non-UUB subsystems. We have $K^{-}(k)+K^{+}(k)=k$.

Similarly, define the Lyapunov function candidate as

$$
V(x[k])=\Psi_{\sigma(k)}(x[k])
$$

For any $k$ satisfying $k_{0}=0<\cdots<k_{i} \leq k<k_{i+1}$, we obtain

$$
V(x[k]) \leq \max \left\{\lambda_{q_{i}}^{k-k_{i}} \Psi_{q_{i}}\left(x\left[k_{i}\right]\right), 1\right\}
$$

Let us define $\lambda_{s}=\max _{1 \leq i \leq r}\left\{\lambda_{q_{i}}\right\}<1$, and $\lambda_{u}=$ $\max _{r<i \leq N}\left\{\lambda_{q_{i}}\right\} \geq 1$. Also, there exists a constant scalar $\mu$ such that $\Psi_{i}(x) \leq \mu \Psi_{j}(x)$ and $\Psi_{j}(x) \leq \mu \Psi_{i}(x)$, for all $x \in \mathbb{R}^{n}$. The scalar $\mu$ can be selected as in the previous section.

Therefore, by induction, we have

$$
V(x[k]) \leq \max \left\{\lambda_{s}^{K^{-(k)}} \lambda_{u}^{K^{+}(k)} \mu^{N_{\sigma}(k)} V(x[0]), 1\right\}
$$

If there exists a positive scalar $0<\lambda<1$ such that

$$
\frac{K^{+}(k)}{k} \leq \frac{\ln \lambda-\ln \lambda_{s}}{\ln \lambda_{u}-\ln \lambda_{s}}
$$

which is a condition on the percentage of time interval that the unstable subsystems are activated, then we obtain

$$
\left(\frac{\lambda_{u}}{\lambda_{s}}\right)^{K^{+}(k)} \leq\left(\frac{\lambda}{\lambda_{s}}\right)^{k} \Leftrightarrow \lambda_{s}^{K^{-}(k)} \lambda_{u}^{K^{+}(k)} \leq \lambda^{k}
$$

And thus

$$
V(x[k]) \leq \max \left\{\lambda^{k} \mu^{N_{\sigma}(k)} V(x[0]), 1\right\} .
$$

Assume that

$$
\lambda^{k} \mu^{N_{\sigma}(k)} \leq\left(\lambda^{*}\right)^{k}
$$


for some $0<\lambda^{*}<1$. This inequality is equivalent to

$$
N_{\sigma}(k) \leq \frac{k}{\tau_{a}^{*}}, \quad \tau_{a}^{*}=\frac{\ln \mu}{\ln \lambda^{*}-\ln \lambda}
$$

which is an average dwell time scheme as well. Therefore

$$
V(x[k]) \leq \max \left\{\left(\lambda^{*}\right)^{k} V(x[0]), 1\right\} .
$$

This implies that the entire system is UUB.

Theorem 2: The switched system (2) achieves a finite asymptotic disturbance attenuation level under switching signals

1) with percentage of time interval that the unstable subsystems are activated less than $\frac{\ln \lambda-\ln \lambda_{s}}{\ln \lambda_{u}-\ln \lambda_{s}}$ in the sense of (11), and

2) with average dwell time no less than $\frac{\ln \mu}{\ln \lambda^{*}-\ln \lambda}$ in the sense of (14).

Compared with the result for all subsystems are UUB, the switching signals in this section have one condition on the percentage of the activation periods of unstable subsystems in addition to a similar average dwell time condition. In other words, the switched system that stays too long in the unstable mode may lead to an unbounded disturbance attenuation performance.

\section{CONCluding Remarks}

In this paper, we investigated the asymptotic disturbance attenuation properties for a class of switched linear systems with parametric uncertainties and exterior disturbances under various switching signals. First, arbitrary switching signals were considered, and a necessary and sufficient condition on the subsystems' dynamics was derived for the switched systems to achieve a finite disturbance attenuation level under the assumption that each subsystem was UUB. In addition, the equivalence between the asymptotic stability of arbitrary switching linear systems and the robust stability of a corresponding linear time-variant systems was obtained, which bridges two originally distinct research fields. Secondly, if the finite disturbance attenuation level is not preserved under arbitrary switch signals, average dwell switching schemes were proposed to attain a finite disturbance attenuation level. Two cases were considered for this problem that depend on whether all the subsystems are UUB or not. The techniques were based on multiple set-induced Lyapunov functions and polyhedral algebra.

\section{REFERENCES}

[1] P. Bauer, K. Premaratne, and J. Duran, "A necessary and sufficient condition for robust asymptotic stability of time-variant discrete systems," IEEE Trans. Automat. Contr., vol. 38, no. 9, pp. 14271430, 1993.

[2] A. A. Agrachev and D. Liberzon, "Lie-algebraic stability criteria for switched systems," SIAM J. Control Optim., vol. 40, pp. 253-270, 2001.

[3] A. Bemporad and M. Morari, "Control of systems integrating logic, dynamics, and constraints," Automatica, vol. 35, no. 3, pp. 407-427, 1999.

[4] A. Bemporad, G. Ferrari-Trecate, and M. Morari, "Observability and controllability of piecewise affine and hybrid systems," IEEE Trans. Automat. Contr., vol. 45, no. 10, pp. 1864-1876, 2000.
[5] F. Blanchini, "Ultimate boundedness control for uncertain discretetime systems via set-induced Lyapunov functions," IEEE Trans. Automat. Contr., vol. 39, no. 2, pp. 428-433, 1994.

[6] F. Blanchini, "Nonquadratic Lyapunov functions for robust control," Automatica, vol. 31, pp. 451-461, 1995.

[7] F. Blanchini, S. Miani, and M. Sznaier, "Robust performance with fixed and worst case signals for uncertain time-varying systems," Automatica, vol. 33, no. 12, pp. 2183-2189, 1997.

[8] W. Dayawansa and C. F. Martin, "A converse Lyapunov theorem for a class of dynamical systems which undergo switching," IEEE Trans. Automat. Contr., vol. 44, no. 4, pp. 751-760, 1999.

[9] R. A. Decarlo, M. S. Branicky, S. Pettersson, and B. Lennartson, "Perspectives and results on the stability and stabilizability of hybrid systems," Proccedings of the IEEE, vol. 88, no. 7, pp. 1069-1082, 2000.

[10] J. P. Hespanha and A. S. Morse, "Stability of switched systems with average dwell-time," in Proceedings of the 38th IEEE Conf. Decision Control, pp. 2655-2660, 1999.

[11] J. P. Hespanha, "Root-mean-square gains of switched linear systems," IEEE Trans. Automat. Contr., vol. 48, no. 11, pp. 2040-2045, 2003.

[12] J. P. Hespanha, "Uniform stability of switched linear systems: Extensions of LaSalle's invariance principle," IEEE Trans. Automat. Contr, vol. 49, no. 4, pp. 470-482, 2004.

[13] M. Johansson, Piecewise Linear Control Systems, Ph.D. Thesis, Lund Institute of Technology, Sweden, 1999.

[14] D. Liberzon and A. S. Morse, "Basic problems in stability and design of switched systems," IEEE Contr. Syst. Magazine, vol. 19, no. 15 , pp. 59-70, 1999.

[15] D. Liberzon, Switching in Systems and Control, Birkhauser, Boston, 2003.

[16] D. Liberzon, J. P. Hespanha and A. S. Morse, "Stability of switched linear systems: A Lie-algebraic condition," Syst. Contr. Lett., vol. 37, no. 3, pp. 117-122, 1999.

[17] D. Liberzon and R. Tempo, "Gradient algorithm for finding common Lyapunov functions," in Proc. 42nd IEEE Conf. Decision Control, pp. 4782-4787, 2003.

[18] H. Lin and P. J. Antsaklis, "Uniformly ultimate boundedness control for uncertain switched linear systems," ISIS Technical Report ISIS2003-004, August 2003.

Available at http://www.nd.edu/ isis/tech.html.

[19] H. Lin and P. J. Antsaklis, "Disturbance attenuation properties for discrete-time uncertain linear switched systems," in Proc. 42nd IEEE Conf. Decision Control, pp. 5289-5294, 2003.

[20] H. Lin and P. J. Antsaklis, "Disturbance attenuation in classes of uncertain linear hybrid systems," in Proc. 2004 American Control Conf., pp. 566-571, 2004.

[21] D. Mignone, G. Ferrari-Trecate, and M. Morari, "Stability and stabilization of piecewise affine and hybrid systems: An LMI approach," in Proc. 39th Conf. Decision Control, pp. 504-509, 2000.

[22] K. S. Narendra and J. Balakrishnan, "A common Lyapunov function for stable LTI systems with commuting A-matrices," IEEE Trans. Automat. Contr., vol. 39, no. 12, pp. 2469-2471, 1994.

[23] R. N. Shorten and K. S. Narendra, "On the stability and existence of common Lyapunov functions for stable linear switching systems," in Proc. 37th IEEE Conf. Decision Control, pp. 3723-3724, 1998.

[24] Z. Sun, S. S. Ge, and T. H. Lee, "Controllability and reachability criteria for switched linear systems," Automatica, vol. 38, no. 5, pp. 775-786, 2002.

[25] M. A. Wicks, P. Peleties, and R. A. DeCarlo, "Construction of piecewise Lyapunov function for stabilizing switched systems," in Proc. 35th IEEE Conf. Decision Control, pp. 3492-3497, 1994.

[26] H. Ye, A. N. Michel, and L. Hou, "Stability theory for hybrid dynamical systems," IEEE Trans. Automat. Contr., vol. 43, no. 4, pp. 461-474, 1998.

[27] G. Zhai, B. Hu, K. Yasuda, and A. N. Michel, "Disturbance attenuation properties of time-controlled switched systems," J. Franklin Institute, vol. 338, pp. 765-779, 2001.

[28] G. Zhai, B. Hu, K. Yasuda, and A. N. Michel, "Qualitative analysis of discrete-time switched systems," in Proc. 2002 American Control Conf., pp. 1880-1885, 2002. 